Louisiana State University

LSU Digital Commons

Faculty Publications

Department of Mathematics

8-1-2008

\title{
On Atkin and Swinnerton-Dyer congruence relations (3)
}

Ling Long

lowa State University

Follow this and additional works at: https://digitalcommons.Isu.edu/mathematics_pubs

\section{Recommended Citation}

Long, L. (2008). On Atkin and Swinnerton-Dyer congruence relations (3). Journal of Number Theory, 128 (8), 2413-2429. https://doi.org/10.1016/j.jnt.2008.02.014

This Article is brought to you for free and open access by the Department of Mathematics at LSU Digital Commons. It has been accepted for inclusion in Faculty Publications by an authorized administrator of LSU Digital Commons. For more information, please contact ir@lsu.edu. 


\title{
On Atkin and Swinnerton-Dyer congruence relations (3)
}

\author{
Ling Long ${ }^{1}$ \\ Department of Mathematics, Iowa State University, Ames, IA 50011, USA \\ Received 10 August 2007; revised 22 January 2008 \\ Available online 8 May 2008 \\ Communicated by David Goss
}

\begin{abstract}
It is now well known that Hecke operators defined classically act trivially on genuine cuspforms for noncongruence subgroups of $S L_{2}(\mathbb{Z})$. Atkin and Swinnerton-Dyer speculated the existence of $p$-adic Hecke operators so that the Fourier coefficients of their eigenfunctions satisfy three-term congruence recursions. In the previous two papers with the same title ([W.C. Li, L. Long, Z. Yang, On Atkin and SwinnertonDyer congruence relations, J. Number Theory 113 (1) (2005) 117-148] by W.C. Li, L. Long, Z. Yang and [A.O.L. Atkin, W.C. Li, L. Long, On Atkin and Swinnerton-Dyer congruence relations (2), Math. Ann. 340 (2) (2008) 335-358] by A.O.L. Atkin, W.C. Li, L. Long), the authors have studied two exceptional spaces of noncongruence cuspforms where almost all $p$-adic Hecke operators can be diagonalized simultaneously or semi-simultaneously. Moreover, it is shown that the $l$-adic Scholl representations attached to these spaces are modular in the sense that they are isomorphic, up to semisimplification, to the $l$-adic representations arising from classical automorphic forms.

In this paper, we study an infinite family of spaces of noncongruence cuspforms (which includes the cases in [W.C. Li, L. Long, Z. Yang, On Atkin and Swinnerton-Dyer congruence relations, J. Number Theory 113 (1) (2005) 117-148; A.O.L. Atkin, W.C. Li, L. Long, On Atkin and Swinnerton-Dyer congruence relations (2), Math. Ann. 340 (2) (2008) 335-358]) under a general setting. It is shown that for each space in this family there exists a fixed basis so that the Fourier coefficients of each basis element satisfy certain weaker three-term congruence recursions. For a new case in this family, we will exhibit that the attached $l$-adic Scholl representations are modular and the $p$-adic Hecke operators can be diagonalized semi-simultaneously.
\end{abstract}

(c) 2008 Elsevier Inc. All rights reserved.

\footnotetext{
E-mail address: linglong@iastate.edu.

1 The author was supported in part by an NSF-AWM mentoring travel grant for women. She would like to thank the Pennsylvania State University and Shan Dong University in China for their hospitalities. During the author's visits to these two institutions this paper was written and revised respectively.
} 


\section{Introduction}

This paper is a continuation of two previous papers with the same title: [LLY05] by W.C. Li, L. Long, Z. Yang and [ALL08] by A.O.L. Atkin, W.C. Li, L. Long. Here, we continue to explore cuspforms for noncongruence subgroups of $S L_{2}(\mathbb{Z})$, which are poorly understood due to the lack of Hecke theory [Tho89,Ber94]. The serious study of these functions was initiated in the late 1960s by Atkin and Swinnerton-Dyer [ASD71] and was further developed by Scholl [Sch85,Sch86,Sch88], etc. In particular, under a general assumption Scholl has established a system of compatible $l$-adic representations of the absolute Galois group attached to each space of noncongruence cuspforms. In [LLY05,ALL08], two intricate cases have been exhibited in which noncongruence cuspforms are related closely to classical congruence cuspforms as follows: From the $p$-adic point of view, in each of these cases there is a simultaneous (or semi-simultaneous) basis for almost all primes $p$ such that each basis function is an eigenfunction for the Hecke operator $T_{p}$ in a $p$-adic sense. Moreover, after multiplying by suitable ideal class characters, the $p$-adic Hecke eigenvalues coincide with Hecke eigenvalues arising from classical newforms. From the representation point of view, in each case the associated $l$-adic Scholl representation $\rho_{l}$ is shown to be isomorphic to an $l$-adic representation attached to a classical congruence automorphic form. Here, we intend to give a more general discussion on the these topics.

In this paper, we will explore cuspforms for character groups (cf. Definition 1). For example, the lattice groups studied by Rankin [Ran67] and all groups in [LLY05,ALL08,KL08] are character groups and most of them are noncongruence. The construction of these groups endows the corresponding modular forms with special arithmetic properties.

This paper is organized in the following way: Section 2 is devoted to the properties of modular forms for character groups. In Section 3, we study the arithmetic properties of weight 3 cuspforms for a special family of noncongruence character groups denoted by $\Gamma_{n}, n \in \mathbb{N}$ (cf. the constructions in Section 3). In particular, we will prove the following theorem.

Theorem 1. Let $n$ be an arbitrary positive integer and $p$ be a prime number which is relatively prime to $5 \cdot n$. Denote by $r$ the order of $p$ in the multiplicative group $(\mathbb{Z} / n \mathbb{Z})^{\times}$. Then the space $S_{3}\left(\Gamma_{n}\right)$ of weight 3 cuspforms for $\Gamma_{n}$ has a basis $\left\{h_{j}=\sum_{m \geqslant 1} a_{j}(m) e^{2 \pi i m z / 5 n}, a_{j}(m) \in \mathbb{Q}\right\}_{j=1}^{n-1}$ independent of $p$. For every $h_{j}$ there exist $A_{j}(p)$ and $B_{j}(p)$ in $\mathbb{Z}\left[e^{2 \pi i / r}\right]$ with $\left|A_{j}(p)\right| \leqslant\left(\begin{array}{c}2 r \\ r\end{array}\right) p^{r}$ and $\left|B_{j}(p)\right|=p^{2 r}$ such that for every positive integer $m$

$$
\frac{a_{j}\left(m p^{r}\right)-A_{j}(p) a_{j}(m)+B_{j}(p) a_{j}\left(m / p^{r}\right)}{(p m)^{2}}
$$

is integral over some place in $\mathbb{Z}\left[e^{2 \pi i / r}\right]$ above $p$. By convention, $a_{j}\left(m / p^{r}\right)=0$ if $p^{r} \nmid m$.

The congruence relation (1) is weaker but more general than those Atkin and Swinnerton-Dyer congruence relations obtained in [LLY05,ALL08].

In Section 4, we investigate the arithmetic properties of weight 3 cuspforms for $\Gamma_{6}$ in the above family. This case is very similar to the one obtained in [ALL08]. The main result is: 
Theorem 2. Let $\rho_{3, l, 6}: \operatorname{Gal}(\overline{\mathbb{Q}} / \mathbb{Q}) \rightarrow \operatorname{Aut}\left(W_{3, l, 6}\right)$ be the l-adic Scholl representations attached to weight 3 cuspforms for $\Gamma_{6}$. Then $\rho_{3, l, 6}$ are isomorphic up to semisimplification to the $l$-adic Galois representations attached to a classical congruence automorphic form. Moreover, for every prime $p \nmid 6$, the space of weight 3 cuspforms has a basis, depending on the congruence class of $p$ modulo 12, such that for each basis element $\sum_{m \geqslant 1} a(m) e^{2 \pi i z m / 30}$ and all $m \in \mathbb{N}$,

$$
\frac{a(m p)-A(p) a(m)+B(p) a(m / p)}{(p m)^{2}}
$$

is integral at some place in $\mathbb{Z}\left[\sqrt{\delta^{\prime}}\right]$ above $p$ where $A(p) \in \mathbb{Z}[\sqrt{\delta}]$ with $|A(p)| \leqslant 2 p$ and $B(p)=$ $\pm p^{2}$. Here $\delta=1,-1,-3,3$ and $\delta^{\prime}=1,-1,-3,-3$ respectively when $p=1,5,7,11$ mod 12 . Moreover, up to multiplying a unit in $\mathbb{Z}[i]$, the $A(p)$ values coincide with the $T_{p}$ Hecke eigenvalues of some weight 3 congruence Hecke eigenform ( $c f .(15))$.

In this paper, let $\Gamma^{0}$ be a congruence subgroup of $P S L_{2}(\mathbb{Z})$ and $\Gamma$ a finite index subgroup of $\Gamma^{0}$. Let $X_{\Gamma}$ denote the compactified modular curve for $\Gamma$. Here we only consider groups whose modular curves are defined over $\mathbb{Q}$. Let $\mathfrak{M}_{\Gamma}$ denote the field of meromorphic modular functions for $\Gamma$. Then, $\mathfrak{M}_{\Gamma}$ is a finite extension of $\mathfrak{M}_{\Gamma^{0}}$. Let $k \geqslant 2$ be an integer. By $S_{k}(\Gamma)$ we mean the space of weight $k$ holomorphic cuspforms for $\Gamma$. In the sequel, let $\omega_{n}=e^{2 \pi i / n}$ and $L_{n}=\mathbb{Q}\left(\omega_{n}\right)$. For any field $K$, let $G_{K}=\operatorname{Gal}(\bar{K} / K)$, where $\bar{K}$ is an algebraic closure of $K$. Unless otherwise mentioned, we follow all other notation used in [ALL08].

\section{Cuspforms for special character groups}

The discussion in this section applies to modular curves defined over $\mathbb{Q}$ of any genus which satisfy a special symmetry stated in Section 2.3.

\subsection{Character groups}

Definition 1. Let $\Gamma^{0}$ be a congruence subgroup of $P S L_{2}(\mathbb{Z})$. A finite index subgroup $\Gamma$ of $\Gamma^{0}$ is called a character group of $\Gamma^{0}$ if it is normal in $\Gamma^{0}$ with abelian quotient.

\subsection{Character groups with cyclic $\Gamma^{0} / \Gamma$}

By the Fundamental Theorem of Finite Abelian Groups, we will focus on character groups with $\Gamma^{0} / \Gamma$ cyclic in the sequel and we assume $\Gamma^{0} / \Gamma=\langle\zeta \Gamma\rangle \cong \mathbb{Z}_{n}$ where $n=\left[\Gamma^{0}: \Gamma\right]$. By the representation theory of finite cyclic groups,

$$
S_{k}(\Gamma)=\bigoplus_{1 \leqslant j \leqslant n} S_{k}\left(\Gamma^{0}, \chi^{j}\right),
$$

where $\chi(\zeta \Gamma)=\omega_{n}, \omega_{n}=e^{2 \pi i / n}$, and $\chi^{j}(1 \leqslant j \leqslant n)$ are the characters of $\Gamma^{0} / \Gamma$. Here

$$
S_{k}\left(\Gamma^{0}, \chi^{j}\right)=\left\{f \in S_{k}(\Gamma)|f|_{\zeta}=\omega_{n}^{j} f\right\},
$$

and "|" denotes the usual stroke operator with respect to weight $k$. When $(j, n)>1, f \in$ $S_{k}\left(\Gamma^{0}, \chi^{j}\right)$ is a cuspform for an intermediate group $\Gamma^{\prime}$ such that $\Gamma \subsetneq \Gamma^{\prime} \subseteq \Gamma^{0}$. Thus, 
$\bigoplus_{(j, n)>1} S_{k}\left(\Gamma^{0}, \chi^{j}\right)$ consists of "old" forms for supergroups of $\Gamma$; while $\bigoplus_{(j, n)=1} S_{k}\left(\Gamma^{0}, \chi^{j}\right)$ consists of "new" forms orthogonal to those "old" forms under the Petersson inner product. Denote these two spaces by $S_{k}(\Gamma)^{\text {old }}$ and $S_{k}(\Gamma)^{\text {new }}$ respectively and we have

$$
S_{k}(\Gamma)=S_{k}(\Gamma)^{\text {new }} \oplus S_{k}(\Gamma)^{\text {old }} .
$$

\subsection{Special symmetry}

Since $\zeta$ normalizes $\Gamma$, it induces an order $n$ cyclic covering map from the modular curve $X_{\Gamma}$ of $\Gamma$ to the modular curve $X_{\Gamma^{0}}$ of $\Gamma^{0}$. Denote by $\zeta$ again the morphism induced by $\zeta$ on $X_{\Gamma}$ and assume its minimal field of definition is $\mathbb{Q}\left(\omega_{n}\right)$. We further assume that as a simple finite extension of $\mathfrak{M}_{\Gamma^{0}}, \mathfrak{M}_{\Gamma}$ is generated by $t_{n}$ satisfying (1) $\left(t_{n}\right)^{n}-t$ for some $t \in \mathfrak{M}_{\Gamma^{0}}$; (2) $t_{n}$ has rational Fourier coefficients at infinity. Then $X_{\Gamma}$ admits a natural automorphism

$$
\zeta: t_{n} \mapsto \omega_{n}^{-1} t_{n}
$$

Such a map $\zeta$ is defined over $L_{n}=\mathbb{Q}\left(\omega_{n}\right)$. Note that in general, the coefficients of $t_{n}$ are in some number field in stead of $\mathbb{Q}$ (cf. the cases in [KL08, Section 6]).

\section{4. -Adic Scholl representations}

Assume $\operatorname{dim}_{\mathbb{C}} S_{k}(\Gamma)=d$. For any prime number $l$, let $\rho_{k, l}: G_{\mathbb{Q}} \rightarrow \operatorname{Aut}\left(W_{k, l}\right)$ be the $l$-adic Scholl representation attached to $S_{k}(\Gamma)$ which is unramified outside of a few primes including $l$ [Sch85]. Here $W_{k, l}$ is a $2 d$-dimensional $\mathbb{Q}_{l}$-vector space. Denoted by $N$ the product of all ram-

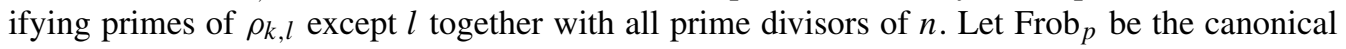
Frobenius conjugacy class in the quotient of the decomposition group at $p$ by the inertia group at $p$. Scholl has shown that the characteristic polynomial of $\rho_{k, l}$ (Frob $p$ ) for any $p \nmid N l$ has integer coefficients and the eigenvalues have the same absolute value $p^{(k-1) / 2}$.

When $\Gamma$ is a character group as above, the map $\zeta$ (3) endows $W_{k, l} \otimes_{\mathbb{Q}_{l}} \mathbb{Q}_{l}\left(\omega_{n}\right)$ with an order $n$ morphism, which is denoted by $\zeta^{*}$ here. Under our assumptions, $W_{k, l} \otimes_{\mathbb{Q}_{l}} \mathbb{Q}_{l}\left(\omega_{n}\right)$ decomposes into eigenspaces of $\zeta^{*}$. Let $\mathcal{W}_{j}$ be such an eigenspace with eigenvalue $\omega_{n}^{j}$ and

$$
W_{k, j}^{\text {new }}:=\bigoplus_{(j, n)=1} \mathcal{W}_{j} \quad \text { and } \quad W_{k, j}^{\text {old }}:=\bigoplus_{(j, n)>1} \mathcal{W}_{j}
$$

The space $W_{k, l}^{\text {new }}$ is simply denoted by $W^{\text {new }}$ when there is no ambiguity.

Lemma 3. Assume $\rho_{k, l}$ is unramified at p. Then as mappings on $W_{k, l}$

$$
\zeta^{*} \rho_{k, l}\left(\operatorname{Frob}_{p}\right)=\rho_{k, l}\left(\operatorname{Frob}_{p}\right)\left(\zeta^{*}\right)^{p}
$$

Proof. Let $F_{p}$ be the Frobenius morphism $x \rightarrow x^{p}$ over $\mathbb{F}_{p}$. As morphisms modulo $p$ on the elliptic modular surface associated with $\Gamma$, we have

$$
F_{p} \zeta\left(t_{n}\right)=F_{p}\left(\omega_{n}^{-1} t_{n}\right)=\omega_{n}^{-p} t_{n}^{p}=\zeta^{p} F_{p}\left(t_{n}\right)
$$

On cohomology, one has the claimed identity. 
For any $p \nmid N l$ and $w \in \mathcal{W}_{j}$,

$$
\zeta^{*}\left(\rho_{k, l}\left(\operatorname{Frob}_{p}\right) w\right)=\rho_{k, l}\left(\operatorname{Frob}_{p}\right)\left(\zeta^{*}\right)^{p} w=\omega_{n}^{j p}\left(\rho_{k, l}\left(\operatorname{Frob}_{p}\right) w\right)
$$

Thus $\rho_{k, l}\left(\right.$ Frob $\left._{p}\right) \mathcal{W}_{j} \subseteq \mathcal{W}_{j p}$. By iteration, $\rho_{k, l}\left(\operatorname{Frob}_{p}\right)$ permutes $\mathcal{W}_{j}$ and

$$
\rho_{k, l}\left(\operatorname{Frob}_{p}\right) \mathcal{W}_{j}=\mathcal{W}_{j p}
$$

Since the group generated by all $\rho_{k, l}\left(\right.$ Frob $\left._{p}\right)$ with $p \nmid N l$ acts on $\left\{\mathcal{W}_{j}\right\}_{j \in(\mathbb{Z} / n \mathbb{Z})^{\times}}$transitively,

$$
\operatorname{dim}_{\mathbb{Q}_{l}\left(\omega_{n}\right)} \mathcal{W}_{j}=\delta_{p}
$$

where $\delta_{p}$ is an integer independent of $j$ in $(\mathbb{Z} / n \mathbb{Z})^{\times}$. Therefore

$$
\operatorname{dim}_{\mathbb{Q}_{l}\left(\omega_{n}\right)} W^{\text {new }}=\delta_{p} \cdot \phi(n),
$$

where $\phi(n)$ is the Euler phi number of $n$. For any $p \nmid n$, use $O_{n}(p)$ to denote the order of $p$ in the multiplicative group $(\mathbb{Z} / n \mathbb{Z})^{\times}$. Let $r=O_{n}(p)$. It is easy to see that

$$
\mathcal{L}_{j, p}:=\bigoplus_{m=1}^{r} \mathcal{W}_{j p^{m}}
$$

is invariant under $\rho_{k, l}\left(\operatorname{Frob}_{p}\right)$.

Corollary 4. The spaces $W_{k, l}^{\text {new }}$ and $W_{k, l}^{\text {old }}$ are invariant under each $\rho_{k, l}\left(\right.$ Frob $\left._{p}\right)$ when $p \nmid N l$. Therefore as a $\operatorname{Gal}(\overline{\mathbb{Q}} / \mathbb{Q})$-module, $W_{k, l} \otimes_{\mathbb{Q}_{l}} \mathbb{Q}_{l}\left(\omega_{n}\right)=W_{k, l}^{\text {new }} \oplus W_{k, l}^{\text {old }}$.

Proof. For any $p \nmid N l,(p, n)=1$, and $(j, n)=(j p, n)$. The assertions follow naturally.

In the remaining part of this section, we will focus on $W_{k, l}^{\text {new }}$ and let $\rho_{k, l}^{\text {new }}: G_{\mathbb{Q}} \rightarrow \operatorname{Aut}\left(W_{k, l}^{\text {new }}\right)$ be the corresponding Galois representation.

Lemma 5. Let $p \nmid N l$ be a prime and $r=O_{n}(p)$. Then

$$
\operatorname{Char}\left(\mathcal{L}_{j, p}, \rho_{k, l}\left(\operatorname{Frob}_{p}\right)\right)(\lambda)=\operatorname{Char}\left(\mathcal{W}_{j}, \rho_{k, l}\left(\operatorname{Frob}_{p}\right)^{r}\right)\left(\lambda^{r}\right)
$$

Proof. We first extend the scalar field to include $\omega_{n}$ and pick $v_{1}, \ldots, v_{\delta_{p}}$ a basis for $\mathcal{W}_{j}$. Hence $\rho_{k, l}\left(\operatorname{Frob}_{p}\right)^{m}\left(v_{1}\right), \ldots, \rho_{k, l}\left(\operatorname{Frob}_{p}\right)^{m}\left(v_{\delta_{p}}\right)$ is a basis for $\mathcal{W}_{j p^{m}}$. Letting $m$ ranging from 0 to $r-1$, we form the following basis

$$
\left\{v_{1}, \ldots, v_{\delta_{p}}, \ldots, \rho_{k, l}\left(\operatorname{Frob}_{p}\right)^{r-1}\left(v_{1}\right), \ldots, \rho_{k, l}\left(\operatorname{Frob}_{p}\right)^{r-1}\left(v_{\delta_{p}}\right)\right\}
$$


for $\mathcal{L}_{j, p}$, the matrix of $\rho_{k, l}\left(\operatorname{Frob}_{p}\right)$ restricted to $\mathcal{L}_{j, p}$ is of the form

$$
F=\left(\begin{array}{ccccc}
0 & \mathrm{I}_{\delta_{p}} & 0 & \cdots & 0 \\
0 & 0 & \mathrm{I}_{\delta_{p}} & \cdots & 0 \\
\vdots & \vdots & \vdots & \ddots & \vdots \\
0 & 0 & 0 & \cdots & \mathrm{I}_{\delta_{p}} \\
E & 0 & 0 & \cdots & 0
\end{array}\right)
$$

where $E=\left.\left(\rho_{k, l}\left(\operatorname{Frob}_{p}\right)\right)^{r}\right|_{\mathcal{W}_{j}}$ is the matrix of $\rho_{k, l}\left(\operatorname{Frob}_{p}\right)^{r}$ restricted to $\mathcal{W}_{j}$. Here we use $\mathrm{I}_{m}$ to denote the rank $m$ identity matrix. Now we compute $\operatorname{det}\left(\lambda \mathrm{I}_{r \delta_{p}}-F\right)$. Eliminating the diagonal blocks $\lambda \mathrm{I}_{\delta_{p}}$ recursively by using the blocks $-\mathrm{I}_{\delta_{p}}$ on the right and then eliminating the block matrices underneath the $-\mathrm{I}_{\delta_{p}}$ blocks, we have

$$
\operatorname{det}\left(\begin{array}{ccccc}
\lambda \mathrm{I}_{\delta_{p}} & -\mathrm{I}_{\delta_{p}} & 0 & \cdots & 0 \\
0 & \lambda \mathrm{I}_{\delta_{p}} & -\mathrm{I}_{\delta_{p}} & \cdots & 0 \\
0 & 0 & \lambda \mathrm{I}_{\delta_{p}} & \cdots & 0 \\
\vdots & \vdots & \vdots & \ddots & \vdots \\
-E & 0 & 0 & \cdots & \lambda \mathrm{I}_{\delta_{p}}
\end{array}\right)=\operatorname{det}\left(\begin{array}{cc}
0 & -\mathrm{I}_{(r-1) \delta_{p}} \\
\lambda^{r} \mathrm{I}_{\delta_{p}}-E & 0
\end{array}\right)
$$

Thus, $\operatorname{det}\left(\lambda \mathrm{I}_{r \delta_{p}}-\left.\rho_{k, l}\left(\operatorname{Frob}_{p}\right)\right|_{\mathcal{L}_{j, p}}\right)=\operatorname{det}\left(\lambda^{r} \mathrm{I}_{\delta_{p}}-\left.\rho_{k, l}\left(\operatorname{Frob}_{p}\right)^{r}\right|_{\mathcal{W}_{j}}\right)$.

Proposition 6. The polynomial $\operatorname{Char}\left(W^{\text {new }}, \rho_{k, l}^{\text {new }}\left(\operatorname{Frob}_{p}\right)\right)(\lambda)$ is in $\mathbb{Z}\left[\lambda^{r}\right]$ with all roots of the same absolute value $p^{(k-1) / 2}$.

Proof. For all $m \mid n$, let $\Gamma_{m}$ denote the unique intermediate group sitting between $\Gamma$ and $\Gamma^{0}$ such that $\Gamma^{0} / \Gamma_{m} \cong \mathbb{Z}_{m}$ and $W_{m, k, l}$ be the $l$-adic Scholl representation space associated with $S_{k}\left(\Gamma_{m}\right)$. Similarly, we let $W_{m, k, l}^{\text {new }}$ denote the subspace of $W_{m, k, l}$ corresponding to "new" forms for $\Gamma_{m}$. By our assumption, $X_{\Gamma_{m}}$ has a model defined over $\mathbb{Q}$.

By Scholl's Theorem [Sch85, 5.3] which says $\operatorname{Char}\left(W_{k, l}, \rho_{k, l}\left(\operatorname{Frob}_{p}\right)\right)(\lambda) \in \mathbb{Z}[\lambda]$ and its eigenvalues have the same absolute value, it suffices to show

$$
\operatorname{Char}\left(W^{\text {new }}, \rho_{k, l}^{\text {new }}\left(\operatorname{Frob}_{p}\right)\right)(\lambda) \in \mathbb{Z}[\lambda]
$$

This can be obtained by applying the second principle of mathematical induction since

$$
W_{k, l}^{\text {old }}=\bigoplus_{m \mid n, 1 \leqslant m<n}\left(\bigoplus_{j,(j, m)=1} \mathcal{W}_{j \cdot \frac{n}{m}}\right)=\bigoplus_{m \mid n, 1 \leqslant m<n} W_{m, k, l}^{\text {new }}
$$

and hence $\operatorname{Char}\left(W_{k, l}^{\text {old }}, \rho_{k, l}^{\text {old }}\left(\operatorname{Frob}_{p}\right)\right)(\lambda) \in \mathbb{Z}[\lambda]$, which implies (7). 


\subsection{Induced representations}

As a consequence of the above discussions we have for any $p \nmid N l$

$$
\operatorname{Tr}\left(\rho_{k, l}^{\text {new }}\left(\operatorname{Frob}_{p}\right)\right)=0 \quad \text { if } p \neq 1 \bmod n .
$$

Since the images of all Frobenius elements will determine any Galois representation up to semisimplification, this implies that for any Dirichlet character $\psi$ of conductor divisible by $n$

$$
\operatorname{Tr}\left(\rho_{k, l}^{\text {new }}\right)=\operatorname{Tr}\left(\rho_{k, l}^{\text {new }} \otimes \psi\right)
$$

For any $j \in(\mathbb{Z} / n \mathbb{Z})^{\times}$, let $\mathcal{W}_{j}$ as before, $L_{n}=\mathbb{Q}\left(\omega_{n}\right)$, and $\rho_{j}: G_{L_{n}} \rightarrow \operatorname{Aut}\left(\mathcal{W}_{j}\right)$ be the corresponding Galois representation. The group $G_{L_{n}}$ is an index- $\phi(n)$ subgroup of $G_{\mathbb{Q}}$ and $\mathcal{W}_{j}$ is a $\delta_{p}$-dimensional representation space of $G_{L_{n}}$. Moreover, the subgroup in $G_{\mathbb{Q}}$ consisting of elements which leave the space $\mathcal{W}_{j}$ invariant is $G_{L_{n}}$ and $G_{\mathbb{Q}}$ permutes $\left\{\mathcal{W}_{j}\right\}_{j \in(\mathbb{Z} / n \mathbb{Z})^{\times}}$transitively. Applying Proposition 19 of [Ser77], we obtain the following result:

Proposition 7. Let $j \in(\mathbb{Z} / n \mathbb{Z})^{\times}$. The representation $\rho_{k, l}^{\text {new }}$ is induced from $\rho_{j}$. Namely

$$
\rho_{k, l}^{\text {new }}=\operatorname{Ind}_{G_{\mathbb{Q}\left(\omega_{n}\right)}}^{G_{\mathbb{Q}}} \rho_{j}
$$

\section{6. $p$-Adic spaces and Atkin and Swinnerton-Dyer type congruences}

Let $S_{k}\left(\Gamma, \mathbb{Z}_{p}\right)=S_{k}(\Gamma) \otimes \mathbb{Z}_{p}$, and $V$ be the $p$-adic Scholl space attached to $S_{k}(\Gamma)$ with $S_{k}\left(\Gamma, \mathbb{Z}_{p}\right)$ as a subspace [Sch85]. These $p$-adic spaces are endowed with the action of the Frobenius morphism, which is denoted by $F$. The map $\zeta$ (3) also induces an order $n$ morphism on $V$ which is denoted by $\zeta^{*}$. Like before, we can define $V_{j}$ to be the $\omega_{n}^{j}$-eigenspace of $\zeta^{*}$ on $V$ (over $\mathbb{Q}_{p}\left(\omega_{n}\right)$ ). Spaces $V^{\text {new }}$ and $V^{\text {old }}$ can be defined in a similar manner.

In [ASD71], Atkin and Swinnerton-Dyer observed that some noncongruence cuspforms satisfy three-term Hecke like recursions in a $p$-adic sense. Their observations have been verified by Cartier for weight 2 cases [Car71] and Scholl [Sch85] for 1-dimensional cases. Here, we will first define Atkin and Swinnerton-Dyer type congruence relations.

Definition 2. Let $K$ be a number field and $f=\sum_{m \geqslant 1} a(m) q_{\mu}^{m}$, where $q_{\mu}=e^{2 \pi i z / \mu}$ for some $\mu \in \mathbb{N}$ be a weight $k$ cuspform. The function $f$ is said to satisfy the Atkin and Swinnerton-Dyer type congruence relation at $p$ with characteristic polynomial $T^{2 d}+A_{1} T^{2 d-1}+\cdots+A_{2 d} \in K[T]$ if for all $m \in \mathbb{N}$,

$$
\frac{a\left(m p^{d}\right)+A_{1} a\left(m p^{d-1}\right)+\cdots+A_{d} a(m)+\cdots+A_{2 d} a\left(m / p^{d}\right)}{(m p)^{k-1}}
$$

belongs to $K$ and is integral at some place in the ring of integers of $K$ above the prime number $p$.

2.7. Atkin and Swinnerton-Dyer type congruences satisfied by cuspforms for noncongruence character groups

Applying Theorem 5.6 in [Sch85], we have 
Theorem 8 (Scholl). For any prime $p \nmid N l$.

$$
\operatorname{Char}(V, F)(T)=\operatorname{Char}\left(W, \rho_{k, l}\left(\operatorname{Frob}_{p}\right)\right)(T) \in \mathbb{Z}[T] .
$$

Proposition 9. (See Fang et al. [ $\left.F^{+} 05\right]$.) Let $G$ be a finite group of automorphisms of the elliptic modular surface $\pi: \mathcal{E}_{\Gamma} \rightarrow X_{\Gamma}$ associated with $\Gamma$. Let $\chi$ be an irreducible character of $G$ and if $V$ is a representation of $G$, let $V^{\chi}$ denote the $\chi$-isotypical subspace of $V$. Let $K / \mathbb{Q}$ be the field of definition of the representation whose character is $\chi$ and let $\lambda$ be a place of $K$ above $l$ and $\wp$ be a place of $K$ above $p$. Finally, we let $r$ to be the smallest positive integer such that $\left(\text { Frob }_{p}\right)^{r} \in G_{K}$. Then

$$
\operatorname{Char}\left(\left(V \otimes K_{\wp}\right)^{\chi}, F^{r}\right)(T)=\operatorname{Char}\left(\left(W \otimes K_{\lambda}\right)^{\chi}, \rho_{k, l}\left(\operatorname{Frob}_{p}\right)^{r}\right)(T)
$$

The proof of this proposition follows from Proposition 4.4 of [Sch85]. In our case, we use $G=$ $\Gamma^{0} / \Gamma$ which is generated by $\zeta \Gamma$. Then $\left(V \otimes K_{\wp}\right)^{\chi^{j}}=V_{j}$ and $\left(W \otimes K_{\lambda}\right)^{\chi^{j}}=\mathcal{W}_{j}$. Consequently, we have

\section{Corollary 10.}

$$
\operatorname{Char}\left(V^{\text {new }}, F\right)(T)=\operatorname{Char}\left(W^{\text {new }}, \rho_{k, l}\left(\operatorname{Frob}_{p}\right)\right)(T) \in \mathbb{Z}[T] .
$$

If $j \in(\mathbb{Z} / n \mathbb{Z})^{\times}$and $r=O_{n}(p)$, then $\operatorname{dim}_{\mathbb{Q}_{p}\left(\omega_{n}\right)} V_{j}=\delta_{p}$ where $\delta_{p}$ is defined as before. We have

$$
\operatorname{Char}\left(\bigoplus_{i=0}^{r-1} V_{j p^{m}}, F\right)(T)=\operatorname{Char}\left(\mathcal{L}_{j, p}, \rho_{k, l}\left(\operatorname{Frob}_{p}\right)\right)(T) \in L_{n}\left[T^{r}\right]
$$

Proposition 11. Let $\Gamma$ is a character group of $\Gamma^{0}$ with $\Gamma^{0} / \Gamma=\langle\zeta \Gamma\rangle \cong \mathbb{Z}_{n}$. Assume $X_{\Gamma}$ has a model defined over $\mathbb{Q}$, and the action of $\zeta$ on $X_{\Gamma}$ is defined over $\mathbb{Q}\left(\omega_{n}\right)$. Let $p \nmid N l$ be a prime, $j \in(\mathbb{Z} / n \mathbb{Z})^{\times}$, and $r=O_{n}(p)$. Assume the space $V_{j}$ has a basis consisting of forms with Fourier coefficients in $L_{n}$. Let $H_{j}(T)=\operatorname{Char}\left(\mathcal{L}_{j, p}, \rho_{k, l}\left(\operatorname{Frob}_{p}\right)\right)(T) \in L_{n}\left[T^{r}\right]$. For every $f \in V_{j}$ with Fourier coefficients in $L_{n}, f$ satisfies Atkin and Swinnerton-Dyer type congruences at $p$ with characteristic polynomial $H_{j}(T)$.

Proof. This follows from Scholl's arguments in [Sch85].

In particular, when $\delta_{p}=2$, we have

$$
H_{j}(T)=T^{2 r}-A_{j}(p) T^{r}+B_{j}(p)
$$

where $\left|A_{j}(p)\right| \leqslant\left(\begin{array}{c}2 r \\ r\end{array}\right) p^{(k-1) r / 2},\left|B_{j}(p)\right|=p^{(k-1) r}$ since the roots of $H_{j}(T)$ have the same absolute value $p^{(k-1) / 2}$. These congruence recursions are weaker than the original three-term Atkin and Swinnerton-Dyer congruence relations when $r>1$.

\section{Atkin and Swinnerton-Dyer type congruences satisfied by weight 3 cuspforms for $\Gamma_{n}$}

In this section, we will use the results obtained in the previous section to derive three-term Atkin and Swinnerton-Dyer type congruences satisfied by weight 3 cuspforms for $\Gamma_{n}$. 


\subsection{The groups $\Gamma_{n}$}

In [LLY05,ALL08], the following family of noncongruence character group is considered. Let

$$
\Gamma^{1}(5)=\left\{\gamma \in S L_{2}(\mathbb{Z}) \mid \gamma \equiv\left(\begin{array}{ll}
1 & 0 \\
* & 1
\end{array}\right) \bmod 5\right\} / \pm \mathrm{I}_{2} .
$$

It is an index-12 congruence subgroup of $S L_{2}(\mathbb{Z})$ with 4 cusps $\infty,-2,0,-5 / 2$. This group has four generators $\gamma_{\infty}, \gamma_{-2}, \gamma_{0}, \gamma_{-5 / 2}$ (each stabilizes one cusp as indicated by the subscripts) subject to one relation $\gamma_{\infty} \gamma_{-2} \gamma_{0} \gamma_{-5 / 2}=\mathrm{I}_{2}$. Let $\varphi_{n}: \Gamma^{1}(5) \rightarrow \mathbb{C}^{\times}$be the homomorphism such that $\varphi_{n}\left(\gamma_{\infty}\right)=\omega_{n}, \varphi_{n}\left(\gamma_{-2}\right)=\omega_{n}^{-1}$, and $\varphi_{n}\left(\gamma_{0}\right)=1=\varphi_{n}\left(\gamma_{-5 / 2}\right)$. The kernel $\Gamma_{n}$ of $\varphi_{n}$ is an index $n$ character group of $\Gamma^{1}(5)$. When $n \neq 1,5, \Gamma_{n}$ is noncongruence [Lon07, Section 5].

Let $E_{1}, E_{2}$ be the weight 3 Eisenstein series for $\Gamma^{1}(5)$ as in [LLY05]. A Hauptmodul for $\Gamma^{1}(5)$ is $t=\frac{E_{1}}{E_{2}}$ which generates $\mathfrak{M}_{\Gamma^{1}(5)}$ and $t_{n}=\sqrt[n]{\frac{E_{1}}{E_{2}}}$ is a Hauptmodul for $\Gamma_{n}$. Such a group $\Gamma_{n}$ has two distinguished normalizers in $S L_{2}(\mathbb{Z}): \zeta=\left(\begin{array}{ll}1 & 5 \\ 0 & 1\end{array}\right), A=\left(\begin{array}{ll}2 & -5 \\ 1 & -2\end{array}\right)$. In particular

$$
\left.E_{1}\right|_{A}=E_{2},\left.\quad E_{2}\right|_{A}=-E_{1},\left.\quad t\right|_{A}=-\frac{1}{t} .
$$

We use the following algebraic model for the elliptic modular surface $\mathcal{E}_{\Gamma_{n}}$ associated with the group $\Gamma_{n}$ to investigate the roles of the above operators:

$$
\begin{aligned}
\mathcal{E}_{\Gamma_{n}}: \quad y^{2}= & t_{n}^{n}\left(x^{3}-\frac{1+12\left(t_{n}^{n}-t_{n}^{3 n}\right)+14 t_{n}^{2 n}+t_{n}^{4 n}}{48 t_{n}^{2 n}} x\right. \\
& \left.+\frac{1+18\left(t_{n}^{n}-t_{n}^{5 n}\right)+75\left(t_{n}^{2 n}+t_{n}^{4 n}\right)+t_{n}^{6 n}}{864 t_{n}^{3 n}}\right) .
\end{aligned}
$$

The above model is canonical in the following sense. When $n=1$, it is the algebraic equation for the universal elliptic curve with 5-torsion point structure. When $n>1$, the degree $n$ cyclic covering map $X_{\Gamma_{n}} \rightarrow X_{\Gamma^{1}(5)}$ ramifying only at 0 and infinity is given by $\left(t_{n}\right)^{n}=a \cdot t$ for some $a \in \mathbb{Q}^{\times}$. We can choose $a=1$ and under this assumption, the "c-equations" of [ASD71, Section 2.3] for $\Gamma_{n}$ can be solved with $\mathbb{Q}$ solutions.

Note that the following nice conditions are satisfied:

(1) The modular curves $X_{\Gamma_{n}}$ and $X_{\Gamma^{1}(5)}$ are defined over $\mathbb{Q}$ for any positive integer $n$.

(2) $\mathfrak{M}_{\Gamma_{n}}=\mathbb{C}\left(t_{n}\right), \mathfrak{M}_{\Gamma^{0}}=\mathbb{C}(t)$ where $t_{n}$ and $t$ have rational Fourier coefficients at infinity such that the covering map from $X_{\Gamma_{n}}$ to $X_{\Gamma^{1}(5)}$ is given by $t=\left(t_{n}\right)^{n}$.

(3) The cusp at which the Fourier expansions of all modular forms are expanded is one of the ramification points of the above covering map.

The actions of $A$ and $\zeta$ on $\mathcal{E}_{\Gamma_{n}}$ are:

$$
\begin{aligned}
A\left(x, y, t_{n}\right) & =\left(-x, \frac{y}{t_{n}}, \frac{\omega_{2 n}}{t_{n}}\right), \\
\zeta\left(x, y, t_{n}\right) & =\left(x, y, \omega_{n}^{-1} t_{n}\right) .
\end{aligned}
$$


Therefore, $A$ and $\zeta$ are defined over $\mathbb{Q}\left(\omega_{2 n}\right)$ and $\mathbb{Q}\left(\omega_{n}\right)$ and have order 4 and $n$ respectively. In fact, the " $A$ " map (defined over $\mathbb{Q}$ ) in [LLY05, p. 130] is a specialization of the $A$ map (12).

Using (11) as an elliptic curve over the $t_{n}$-line, one can construct a compatible family of $2(n-1)$-dimensional $l$-adic Galois representations $\rho_{3, l, n}^{*}: G_{\mathbb{Q}} \rightarrow \operatorname{Aut}\left(W_{3, l, n}\right)$ where $W_{3, l, n}$ is constructed via the parabolic cohomology associated with the elliptic fibration (11) in the same way as $W_{n, l}$ constructed in [ALL08, Eq. (7)]. The representation $\rho_{3, l, n}^{*}$ is unramified at every prime not dividing $l \cdot n$. Like [ALL08, Section 3.1], one can show that $\rho^{*}$ are isomorphic to the $l$-adic Scholl representations $\rho_{3, l, n}$ associated with $S_{3}\left(\Gamma_{n}\right)$. Let $\zeta^{*}$ and $A^{*}$ be the maps on $W_{k, l, n}$ induced by $A$ and $\zeta$ on $\mathcal{E}_{n}$.

\subsection{The space $S_{3}\left(\Gamma_{n}\right)$}

Let $n>1, k=3$ and we consider the space $S_{3}\left(\Gamma_{n}\right)$. For any $j \in(\mathbb{Z} / n \mathbb{Z})^{\times}$, we can compute that

$$
\delta_{p}=\operatorname{dim}_{\mathbb{Q}_{l}\left(\omega_{n}\right)} \mathcal{W}_{j}=\operatorname{dim}_{\mathbb{Q}_{p}\left(\omega_{n}\right)} V_{j}=2
$$

For any positive integer $n$, let $\left.h_{j}^{[n]}=\sqrt[n]{E_{1}^{n-j} E_{2}^{j}} \in \mathbb{Z}[1 / n][w]\right], w=e^{2 \pi i z / 5 n}$. By [ALL08, Proposition 1], $S_{3}\left(\Gamma_{n}\right)=\left\langle h_{j}^{[n]}\right\rangle_{j=1}^{n-1}$. It is straightforward to verify that $h_{j}^{[n]} \in V_{j}$. According to Proposition 11 , we have

Theorem 12. For every basis element $h_{j}^{[n]}$ of $S_{3}\left(\Gamma_{n}\right)$ and any prime $p \nmid 5 \cdot n, h_{j}^{[n]}$ satisfies threeterm Atkin and Swinnerton-Dyer type congruence relations at $p$ with characteristic polynomial $\operatorname{Char}\left(\mathcal{W}_{j}, \rho_{k, l}\left(\operatorname{Frob}_{p}\right)^{r}\right)\left(T^{r}\right)$ as in Lemma 5 where $r=O_{n}(p)$.

To achieve this result, we only need the operator $\zeta$. Using the $A$ map in addition, we will obtain the following result.

Let $\rho_{3, l, n}^{\text {new }}: G_{\mathbb{Q}} \rightarrow \operatorname{Aut}\left(W_{3, l, n}^{\text {new }}\right)$ be the $l$-adic representation attached to the cuspforms genuinely belonging to $\Gamma_{n}$ as before. When $n=2,3$, and 4 , let $B$ be $A^{*}, A^{*}$, and $\zeta^{*}$; let $K$ be $\mathbb{Q}(i), \mathbb{Q}(\sqrt{-3})$, and $\mathbb{Q}(i)$ respectively. The action of $B$ on $W_{3, l, n}^{\text {new }}$ satisfies $B^{2}=-\mathrm{id}$. Decompose $W_{3, l, n}^{\text {new }}$ according to the $( \pm i)$-eigenspaces of $B$. As a Galois $G_{K}$-module, $W_{3, l, n}^{\text {new }}=$ $W_{3, l, n, i}^{\text {new }} \oplus W_{3, l, n,-i}^{\text {new }}$. Let $\rho_{3, l, n, i}^{\text {new }}: G_{K} \rightarrow \operatorname{Aut}\left(W_{3, l, n, i}^{\text {new }}\right)$ be the corresponding Galois representation. Applying Proposition 19 of [Ser77], we have

Proposition 13. When $n=2,3,4$,

$$
\rho_{3, l, n}^{\text {new }}=\operatorname{Ind}_{G_{K}}^{G_{\mathbb{Q}}} \rho_{3, l, n, i}^{\text {new }}
$$

Remark 14. The proposition provides a different perspective for the results in [LLY05]. When $n=2, \rho_{3, l, n, i}^{\text {new }}$ is a one-dimensional representation of $G_{\mathbb{Q}(i)}$, hence a character of $G_{\mathbb{Q}(i)}$. Naturally it corresponds to a cuspform $\eta(4 z)^{6}$ with complex multiplication [LLY05, Section 8]. Here $\eta(z)$ is the Dedekind eta function. When $n=3, \rho_{3, l, 3}^{\text {new }}$ is induced from a 2-dimensional representation of $G_{\mathbb{Q}(\sqrt{-3})}$. Such a point of view was suggested by J.P. Serre as one of his comments on [LLY05]. A similar result also holds for the $n=6$ case when another specialization of $A$, called $\widetilde{A}$ (cf. (14)) is used. 


\section{4. $S_{3}\left(\Gamma_{6}\right)$}

\section{1. l-Adic spaces}

When $k=3, \operatorname{dim}_{\mathbb{Q}_{l}\left(\omega_{n}\right)} W_{3, l, n}^{\text {new }}=2 \phi(n)$. Accordingly, $\operatorname{dim}_{\mathbb{Q}_{l}\left(\omega_{n}\right)} W_{3, l, n}^{\text {new }} \leqslant 4$ only when $n=$ 2, 3, 4 or 6. Since the first three cases have been handled in [LLY05,ALL08], we will now treat the case $n=6$ here. The current case under consideration shares a lot of similarities with the case $n=4$ studied in [ALL08]. To avoid duplication, we will refer the readers to [ALL08,LLY05] for some arguments. Here, we use the following automorphism which is a specialization of $A$ :

$$
\widetilde{A}\left(x, y, t_{6}\right)=\left(-x, \frac{y}{t_{6}}, \frac{i}{t_{6}}\right) .
$$

The morphism $\widetilde{A}(14)$ is preferred since it is defined over $\mathbb{Q}(i)$ while $A$ (12) is defined over $\mathbb{Q}\left(e^{2 \pi i / 12}\right)$. Let $\widetilde{A}^{*}$ be the corresponding action on $W_{3, l, n}$.

Associated with $S_{3}\left(\Gamma_{6}\right)$ is a 10-dimensional $l$-adic Scholl representation

$$
\rho_{3, l, 6}: \operatorname{Gal}(\overline{\mathbb{Q}} / \mathbb{Q}) \rightarrow \operatorname{Aut}\left(W_{3, l, 6}\right) .
$$

Like the discussion in [ALL08], one can argue that $\rho_{3, l, 6}$ is unramified at 5 if $l \neq 5$. Therefore, in this case we can pick $N=6$. As before, the space $W_{3, l, 6} \otimes_{\mathbb{Q}_{l}} \mathbb{Q}_{l}\left(\omega_{6}\right)$ decomposes naturally into eigenspaces $\mathcal{W}_{j}$ of $\zeta^{*}$. Similar to the discussion in Section 5.3 of [ALL08], $\mathcal{W}_{3}$ (resp. $\left.\mathcal{W}_{2} \oplus \mathcal{W}_{4}\right)$ is isomorphic to the $l$-adic Scholl representation associated with weight 3 cuspforms for $\Gamma_{2}$ (resp. $\Gamma_{3}$ ) tensoring with $\mathbb{Q}_{l}\left(\omega_{6}\right)$. The remaining piece $\mathcal{W}_{1} \oplus \mathcal{W}_{5}$, denoted by $W_{3, l, 6}^{\text {new }}$ (or simply $W^{\text {new }}$ ), is a $\operatorname{Gal}(\overline{\mathbb{Q}} / \mathbb{Q})$-module (cf. Corollary 4). By Proposition 6 , for prime $p \nmid 6 \cdot l$,

$$
\operatorname{Char}\left(W_{3, l, 6}^{\text {new }}, \rho_{3, l, 6}\left(\operatorname{Frob}_{p}\right)\right)(T) \in \mathbb{Z}[T]
$$

Following the discussions in [ALL08], we can show that for all $p \nmid 6$ the characteristic polynomial of $\rho_{3, l, 6}\left(\right.$ Frob $\left._{p}\right)$ can be computed via the algebraic equation (11). By using a Magma program, we obtain the following list:

Table 1

\begin{tabular}{ll}
\hline$p$ & Char $\left(W^{\text {new }}, \rho_{3, l, 6}\left(\operatorname{Frob}_{p}\right)\right)(T)$ \\
\hline 5 & $\left(T^{2}+7 i T-5^{2}\right)\left(T^{2}-7 i T-5^{2}\right)=T^{4}-T^{2}+5^{4}$ \\
7 & $\left(T^{2}+5 \sqrt{-3} T-7^{2}\right)\left(T^{2}-5 \sqrt{-3} T-7^{2}\right)=T^{4}-23 T^{2}+7^{4}$ \\
11 & $\left(T^{2}-5 \sqrt{-3} T-11^{2}\right)\left(T^{2}+5 \sqrt{-3} T-11^{2}\right)=T^{4}-167 T^{2}+11^{4}$ \\
13 & $\left(T^{2}+20 T+13^{2}\right)^{2}$ \\
17 & $\left(T^{2}+8 i T-17^{2}\right)\left(T^{2}-8 i T-17^{2}\right)=T^{4}-514 T^{2}+17^{4}$ \\
19 & $\left(T^{2}+6 \sqrt{-3} T-19^{2}\right)\left(T^{2}-6 \sqrt{-3} T-19^{2}\right)=T^{4}-614 T^{2}+19^{4}$ \\
23 & $\left(T^{2}-2 \sqrt{-3} T-23^{2}\right)\left(T^{2}+2 \sqrt{-3} T-23^{2}\right)=T^{4}-1046 T^{2}+23^{4}$ \\
37 & $\left(T^{2}+10 T+37^{2}\right)^{2}$ \\
53 & $\left(T^{2}-47 i T-53^{2}\right)\left(T^{2}+47 i T-53^{2}\right)=T^{4}-3409 T^{2}+53^{4}$ \\
\hline
\end{tabular}




\subsection{Modularity of $\rho_{3,2,6}$}

The reader will find the following section resembles Section 8 of [ALL08]. But the modularity is achieved via a method similar to that used in [LLY05]. Moreover, we will focus on the modularity of $\rho_{3, l, 6}^{\text {new }}$ in this section.

The action of $\widetilde{A}$ on $X_{\Gamma_{6}}$ is defined over $\mathbb{Q}(i)$ and on $W^{\text {new }}\left(\widetilde{A}^{*}\right)^{2}=-\mathrm{id}$. When it is restricted to $G_{\mathbb{Q}(i)},\left.\rho_{3, l, 6}^{\text {new }}\right|_{G_{\mathbb{Q}(i)}}$ decomposes into two representations $\rho_{ \pm}^{\text {new }}: G_{\mathbb{Q}(i)} \rightarrow \operatorname{Aut}\left(W_{ \pm}^{\text {new }}\right)$ according to the $( \pm i)$-eigenspaces of $\widetilde{A}^{*}$. Each $W_{ \pm}^{\text {new }}$ is a 2-dimensional $\mathbb{Q}_{l}(i)$-vector space.

We have identified a level $2^{8} 3^{3}$ weight 3 and character $\chi_{-4}$ (the Dirichlet character attached to $\mathbb{Q}(i)$ ) congruence Hecke eigenform by using Magma with the first few terms given as below

$$
\begin{aligned}
g^{\prime}:= & q+7 q^{5}-5 \sqrt{-3} q^{7}+5 \sqrt{-3} q^{11}+20 q^{13}-8 q^{17}+6 \sqrt{-3} q^{19} \\
& -2 \sqrt{-3} q^{23}+24 q^{25}+10 q^{29}-31 \sqrt{-3} q^{31}-35 \sqrt{-3} q^{35}+q^{37} \\
& -10-50 q^{41}+10 \sqrt{-3} q^{43}+50 \sqrt{-3} q^{47}-26 q^{49}-47 q^{53}+\cdots \\
= & \sum_{n \geqslant 1} a(n) q^{n}, \quad q=e^{2 \pi i z} .
\end{aligned}
$$

The Fourier coefficients of $g^{\prime}$ are in the ring $\mathbb{Z}\left[\frac{1+\sqrt{-3}}{2}\right]$. For any place $\lambda$ in $\mathbb{Z}\left[\frac{1+\sqrt{-3}}{2}\right]$, denote by $\rho^{\prime}$ the $\lambda$-adic Deligne representations attached to the newform $g^{\prime}$ and denote by $\rho$ the restriction of $\rho^{\prime}$ to $G_{\mathbb{Q}(i)}$. Let $L=\mathbb{Q}(i, \sqrt[4]{3})$. Like [ALL08, Section 8.1], define an ideal class character $\chi$ by composing the Artin reciprocity map from the idele class group of $\mathbb{Q}(i)$ to $\operatorname{Gal}(L / \mathbb{Q}(i))$ with the isomorphism which sends $\operatorname{Gal}(L / \mathbb{Q}(i))$ to $\langle i\rangle$. In particular,

- $\chi(p)=1$ for any $p=3 \bmod 4$;

- $\chi(p)= \pm 1$ for any prime $v$ above $p$ when $p=1 \bmod 12$, depending on the value of $(3)^{(p-1) / 4} \bmod v$;

- $\chi(p)= \pm i$ for any prime $v$ above $p$ when $p=5 \bmod 12$, depending on the value of $(3)^{(p-1) / 4} \bmod v$.

By the base change of Langlands, the $L$-function of $\rho \otimes \chi$ is

$$
\begin{aligned}
L(s, \rho \otimes \chi)= & \prod_{p \equiv 3 \bmod 4} \frac{1}{\left(1-a(p) p^{-s}-p^{2-2 s}\right)\left(1+a(p) p^{-s}-p^{2-2 s}\right)} \\
& \times \prod_{p \equiv 1 \bmod 12} \prod_{v_{1}, v_{2} \mid p} \frac{1}{\left(1-\chi\left(v_{1}\right) a(p) p^{-s}+p^{2-2 s}\right)\left(1-\chi\left(v_{2}\right) a(p) p^{-s}+p^{2-2 s}\right)} \\
& \times \prod_{p \equiv 5 \bmod 12} \frac{1}{\left(1-a(p) i p^{-s}-p^{2-2 s}\right)\left(1+a(p) i p^{-s}-p^{2-2 s}\right)} .
\end{aligned}
$$

Lemma 15. The representation $\rho \otimes \chi$ can be regarded as a 2-dimensional representation of $G_{\mathbb{Q}(i)}$ over $\mathbb{Q}_{l}(i)$, where $l$ is the prime in $\mathbb{Z}$ below $\lambda$ in $\mathbb{Z}\left[\frac{1+\sqrt{-3}}{2}\right]$.

Proof. From Magma's data we know the coefficients of $g^{\prime}$ are in $\mathbb{Z}\left[\frac{1+\sqrt{-3}}{2}\right]$. Let $p$ is a prime not dividing 6. We first note that $|a(p)| \leqslant 2 p$. When $p=1 \bmod 4(\operatorname{resp} . p=3 \bmod 4)$, the 
characteristic polynomial of $\rho^{\prime}\left(\right.$ Frob $\left._{p}\right)$ is of the form $T^{2}-a(p) T+p^{4}\left(\operatorname{resp} . T^{2}-a(p) T-p^{4}\right)$. This characteristic polynomial has two roots of the same absolute values. As a consequence, $a(p) \in \mathbb{Z}\left[\frac{1+\sqrt{-3}}{2}\right]$ is real (resp. totally imaginary). Hence $a(p) \in \mathbb{Z}($ resp. $a(p) / \sqrt{-3} \in \mathbb{Z})$ when $p=1 \bmod 4(p=3 \bmod 4)$. Therefore, the local $L$-factors over odd primes $\wp$ of $\mathbb{Z}[i]$ are of the form $\left(1-c_{p} N(\wp)^{-s} \pm N(\wp)^{2-2 s}\right)^{-1}$ for some $c_{p} \in \mathbb{Z}[i]$.

As representations, $\rho^{\prime}$ of $G_{\mathbb{Q}}$ is over $\mathbb{Q}_{l}\left(\frac{1+\sqrt{-3}}{2}\right), \rho$ of $G_{\mathbb{Q}(i)}$ is over $\mathbb{Q}_{l}$, and $\rho \otimes \chi$ of $G_{\mathbb{Q}(i)}$ is over $\mathbb{Q}_{l}(i)$.

The remaining discussion in this subsection is devoted to the proof of the following theorem.

Theorem 16. The representation $\rho_{+}^{\text {new }}$ of $G_{\mathbb{Q}(i)}$ is isomorphic, up to semisimplification, to $\rho \otimes \chi$ of $G_{\mathbb{Q}(i)}$ over the field $\mathbb{Q}_{2}(i)$.

Unlike the case in [ALL08], we cannot apply Livné's modularity criterion as the traces are not always even. We will use a special case of the Faltings-Serre's modularity criterion [Ser]. A detailed proof of this criterion is provided in [LLY05] in the discussion after Theorem 6.2.

Since both $\rho_{+}^{\text {new }}$ and $\rho \otimes \chi$ are representations over $\mathbb{Q}_{l}(i)$, we take $l=2$ and $\lambda=1+i$. Then $\mathbb{Z}_{2}(i) / \lambda \mathbb{Z}_{2}(i) \cong \mathbb{F}_{2}$. The ramified places are $1+i$ and 3 .

We now recall Theorem 6.2 of [LLY05].

Theorem 17 (Serre). Let $\rho_{1}$ and $\rho_{2}$ be representations of $\mathrm{Gal}(\overline{\mathbb{Q}} / \mathbb{Q}(i))$ to $G L_{2}\left(\mathbb{Z}[i]_{1+i}\right)$. Assume they satisfy the following two conditions:

(1) $\operatorname{det}\left(\rho_{1}\right)=\operatorname{det}\left(\rho_{2}\right)$;

(2) the two homomorphisms from $\operatorname{Gal}(\overline{\mathbb{Q}} / \mathbb{Q}(i))$ to $G L_{2}\left(\mathbb{F}_{2}\right)$, obtained from the reductions of $\rho_{1}$ and $\rho_{2}$ modulo $1+i$, are surjective and equal.

If $\rho_{1}$ and $\rho_{2}$ are not isomorphic up to semisimplification, then there exists a pair $(\widetilde{G}, t)$, where $\widetilde{G}$ is a quotient of the Galois group $\operatorname{Gal}(\overline{\mathbb{Q}} / \mathbb{Q}(i))$ isomorphic to either $S_{4} \times\{ \pm 1\}$ or $S_{4}$ or $S_{3} \times\{ \pm 1\}$, and the map $t: \widetilde{G} \rightarrow \mathbb{F}_{2}$ has value 0 on the elements of $\widetilde{G}$ of order $\leqslant 3$, and 1 on the other elements.

Lemma 18. As 1-dimensional representations of $G_{\mathbb{Q}(i)}$, $\operatorname{det} \rho_{+}^{\text {new }} \cong \operatorname{det} \rho \otimes \chi$.

Proof. We use the idea of the proof of [LLY05, Lemma 5.2]. The only quadratic extensions of $\mathbb{Q}(i)$ unramified outside of $1+i$ and 3 are $\mathbb{Q}(i)(\sqrt{d})$ where $d=i,(1+i),(1-i), 3,3 i$, $3(1+i), 3(1-i)$. Respectively, $p=3+2 i, 3+2 i, 3-2 i, 4+i, 3+2 i, 4-i, 4+i$ are inert in $\mathbb{Q}(i)(\sqrt{d})$. Hence, it suffices to compare the values of the two characters $\operatorname{det} \rho_{+}^{\text {new }}$ and $\operatorname{det} \rho \otimes \chi$ at primes $3 \pm 2 i$ and $4 \pm i$.

Lemma 19. There is only one representation $\bar{\rho}$ from $G_{\mathbb{Q}(i)}$ to $G L_{2}\left(\mathbb{F}_{2}\right)$ unramified outside of $1+i$ and 3 such that the characteristic polynomials of $\rho\left(\right.$ Frob $\left._{\wp}\right)$ are equal to those given in Table 1 modulo $1+i$. Furthermore $\bar{\rho}$ is surjective.

Proof. Let sgn: $S L_{2}\left(\mathbb{F}_{2}\right) \cong S_{3} \rightarrow\{ \pm 1\}$ be the sign function. The kernel of $\bar{\rho} \circ$ sgn fixes a quadratic field $\mathbb{Q}(i)(\sqrt{d})$ of $\mathbb{Q}(i)$ which is unramified outside of $1+i$ and 3 . The possible 
$d$ 's are $1, i, 1+i, 1-i, 3,3 i, 3+3 i, 3-3 i$. By the assumption, the image of Frob $7 \pm 2 i$ under $\bar{\rho}$ is $T^{2}+T+1$. Hence its image is of order 3 under $\bar{\rho}$ and is 1 under $\bar{\rho} \circ$ sgn. However, when $d=i, 1+i, 1-i, 3,3+3 i, 3-3 i$, either Frob $_{7+2 i}$ or Frob $7-2 i$ is inert in $\mathbb{Q}(i)(\sqrt{d})$. So the only possible choices are $d=1,3 i$.

Now we will show $\bar{\rho}$ is surjective. Otherwise, $d=1$ and the kernel of $\bar{\rho} \circ$ sgn fixes a cyclic cubic Galois extension $M$ of $\mathbb{Q}(i)$ which is unramified outside of $1+i$ and 3. Thus $\operatorname{Gal}(M / \mathbb{Q})$ has order 6. Since the order of $\bar{\rho}\left(\operatorname{Frob}_{7}\right)$ is at least $6, \operatorname{Gal}(M / \mathbb{Q})=\mathbb{Z}_{6}$ where $M=\mathbb{Q}(i)\left(\omega_{9}+\omega_{9}^{-1}\right)$ is the splitting field of $f(x)=x^{3}-3 x+1$ over $\mathbb{Q}(i)$. The polynomial $f(x)$ is irreducible modulo $3+2 i$. Meanwhile, the characteristic polynomial of $\bar{\rho}\left(\right.$ Frob $\left._{3 \pm 2 i}\right)$ is $T^{2}+1$. Its order should be 1 or 2 . This leads to a contradiction.

Finally we assume that $\operatorname{ker}(\bar{\rho} \circ \mathrm{sgn})=\operatorname{Gal}(\overline{\mathbb{Q}} / K)$ for some $S_{3}$-extension of $\mathbb{Q}(i)$ which contains $\mathbb{Q}(i)(\sqrt{3 i})$. Namely, $K$ is the splitting field of an irreducible cubic polynomial $f(x)$ over $\mathbb{Q}(i)$. Among all non-isomorphic order 12 groups, there is only one which contains $S_{3}$ as a subgroup. Therefore, $\operatorname{Gal}(K / \mathbb{Q})$ is isomorphic to $S_{3} \times \mathbb{Z}_{2}$. Hence, we may assume $f(x)=$ $x^{3}+p x+q, p, q \in \mathbb{Z}$, such that the discriminant $\Delta=-4 p^{3}-27 q^{2}= \pm(3 i)(1+i)^{2 \alpha} 3^{2 \beta}$, $\alpha, \beta \in \mathbb{N}$. Since $\Delta \in \mathbb{Z}, \Delta= \pm 6 \cdot s^{2}$ for some $s \in \mathbb{Z}$. Therefore $\mathbb{Q}(\sqrt{-6})$ and $\mathbb{Q}(\sqrt{6})$ are subfields contained in $K$ which implies that $K$ contains an $S_{3}$-extension $K^{\prime}$ of $\mathbb{Q}$ unramified outside of 2 and 3 with an intermediate field $\mathbb{Q}(\sqrt{-6})$. By the discussion in Section 6 of [LLY05], we know such $K^{\prime}$ is unique and is the splitting field of $f(x)=x^{3}+3 x-2$. Hence $K$ is also unique and it is the splitting field of $f$ over $\mathbb{Q}(i)$.

It is easy to see $\rho_{+}^{\text {new }}$ and $\rho \otimes \chi$ modulo $1+i$ satisfy the conditions for $\bar{\rho}$. Consequently, when modulo $1+i$, the representations $\rho_{+}^{\text {new }}$ and $\rho \otimes \chi$ are surjective on $G L_{2}\left(\mathbb{F}_{2}\right)$ and are isomorphic up to semisimplification.

We now find all $S_{4}$-extensions $E$ of $\mathbb{Q}(i)$ which contain $K$ and are unramified away from $(1+i)$ and 3 . There is a unique order 48 group which has a normal subgroup isomorphic to $S_{4}$, which is $S_{4} \times \mathbb{Z}_{2}$. (The group $S_{4} \rtimes \mathbb{Z}_{2}$ is isomorphic to $S_{4} \times \mathbb{Z}_{2}$ since all automorphisms of $S_{4}$ are inner.) Consequently, each $E$ corresponds to a unique $S_{4}$-extension of $\mathbb{Q}$ unramified away from 2 and 3 and containing the splitting field of $f(x)$. All such $S_{4}$-extensions of $\mathbb{Q}$ (as splitting fields of degree 4 polynomials $g(x)$ ) have already been given in Section 6 of [LLY05, p. 138].

\begin{tabular}{ll}
\hline Defining equation $g(x)$ & $v$ with order 4 Frobenius in $G_{E}$ \\
\hline$x^{4}-4 x-3=0$ & $3+2 i$ \\
$x^{4}-8 x+6=0$ & $3+2 i$ \\
$x^{4}-12 x^{2}-16 x+12=0$ & $6+i$ \\
\hline
\end{tabular}

To conclude the claim of Theorem 16, it suffices to compare the local Euler- $p$ factors of $L$ functions attached to the two representations at $p=13,17,37$, and 53 .

At last we exclude the possibility that $\widetilde{G}$ being isomorphic to $S_{3} \times\{ \pm 1\}$ by comparing the two representations at Frob $1 \pm 2 i$. We first list all quadratic extensions $L$ of $\mathbb{Q}(i)$ unramified outside of $1+i$ and 3 such that $L$ is not contained in $K$, the splitting field of $x^{3}+3 x-2$ over $\mathbb{Q}(i)$. So $L=\mathbb{Q}(i)(\sqrt{d})$ where $d=i, 1+i, 1-i, 3,3+3 i, 3-3 i$. Note that $1+2 i, 1+2 i, 1-2 i, 1+2 i$, $1-2 i, 1+2 i$ are inert in $L$ respectively. Since the characteristic polynomials of $\bar{\rho}\left(\right.$ Frob $\left._{1 \pm 2 i}\right)$ are $T^{2}+T+1$, either Frob $1+2 i$ or Frob $1-2 i$ in $\widetilde{G}$ has order 6 .

Hence we have completed the proof of Theorem 16. 


\section{3. $p$-Adic spaces}

Let $V$ be the $p$-adic Scholl space attached to $S_{3}\left(\Gamma_{6}\right)$ with the action of Frobenius $F$. We will drop the superscript [6] below for convenience. Denote also by $\widetilde{A}^{*}$ the action on $V$ induced $\widetilde{A}$. Decompose $V$ accordingly into $V^{\text {old }} \oplus V^{\text {new }}$ such that

$$
h_{2}, h_{3}, h_{4} \in V^{\text {old }} \quad \text { and } \quad V^{\text {new }}=V_{1} \oplus V_{5},
$$

where $h_{1} \in V_{1}$, and $h_{5} \in V_{5}$.

By considering $h_{j}=E_{2} \cdot t_{n}^{n-j}$ and the actions of $\zeta^{*}$ and $\widetilde{A}^{*}$ on $E_{1}, E_{2}$, and $t_{n}$, we obtain

$$
\zeta^{*}\left(h_{j}\right)=\left.h_{j}\right|_{\zeta}=\omega_{n}^{j} h_{j}, \quad \widetilde{A}^{*}\left(h_{j}\right)=\left.h_{j}\right|_{\widetilde{A}}=-i^{n-j} h_{n-j} .
$$

It follows

$$
\zeta^{*} \widetilde{A}^{*} \zeta^{*}=\widetilde{A}^{*}
$$

A straightforward computation reveals that

$$
(F)^{-1} \widetilde{A}^{*} F=\widetilde{A}^{*}\left(\zeta^{*}\right)^{3(1-p) / 2}
$$

\subsection{The three-term Atkin and Swinnerton-Dyer congruences}

In this case $(F)^{-1} \widetilde{A}^{*} F=\widetilde{A}^{*}\left(\zeta^{*}\right)^{3(1-p) / 2}$ and $\zeta^{*} F=F\left(\zeta^{*}\right)^{p}$. Let

$$
B_{-1}=\widetilde{A}^{*}, \quad B_{-3}=\left(\zeta^{*}-\left(\zeta^{*}\right)^{-1}\right), \quad B_{3}=\left(\zeta^{*}-\left(\zeta^{*}\right)^{-1}\right) \widetilde{A}^{*} .
$$

When $p=5,7,11$ modulo $12, B_{-1}, B_{-3}$, and $B_{3}$ commute with $F$ and are defined over $\mathbb{Q}_{p}$ respectively. Moreover on $V^{\text {new }}$,

$$
\begin{gathered}
\left(\widetilde{A}^{*}\right)^{2}=-\mathrm{id}, \\
\left(\zeta^{*}-\left(\zeta^{*}\right)^{-1}\right)^{2}=\left(\zeta^{*}\right)^{2}+\left(\zeta^{*}\right)^{-2}-2 \mathrm{id}=-3 \mathrm{id}, \\
\left(\left(\zeta^{*}-\left(\zeta^{*}\right)^{-1}\right) A^{*}\right)^{2}=\left(\zeta^{*}-\left(\zeta^{*}\right)^{-1}\right)\left(\left(\zeta^{*}\right)^{-1}-\zeta^{*}\right)\left(A^{*}\right)^{2}=-3 \mathrm{id} .
\end{gathered}
$$

Hence

$$
B_{-1}^{2}=-\mathrm{id}, \quad B_{-3}^{2}=B_{3}^{2}=-3 \mathrm{id}
$$

Now apply the analysis in Sections 6 and 7 of [ALL08] with the roles of $B_{ \pm 2}$ replaced by $B_{ \pm 3}$ and $A$ replaced by $\widetilde{A}$, we obtain the following theorem:

Theorem 20 (Atkin and Swinnerton-Dyer congruence for $S_{3}\left(\Gamma_{6}\right)$ ). The Atkin and SwinnertonDyer congruence holds on the space $S_{3}\left(\Gamma_{6}\right)=\left\langle h_{1}, h_{2}, h_{3}, h_{4}, h_{5}\right\rangle$. More precisely, $h_{3}$ lies in $\mathrm{S}_{3}\left(\Gamma_{2}\right)$ and it satisfies the Atkin and Swinnerton-Dyer congruence relations with the weight 3 congruence newform $g_{2}(z)=\eta(4 z)^{6} ; h_{2}, h_{4} \in S_{3}\left(\Gamma_{3}\right)$ and $h_{2} \pm i h_{4}$ satisfy Atkin and SwinnertonDyer congruence relations with two explicit level 27 congruence newforms respectively (cf. 
[LLY05]). For each prime $p \nmid 6$, the subspace $\left\langle h_{1}, h_{5}\right\rangle$ has a basis depending on the residue of $p$ modulo 12 satisfying 3-term Atkin and Swinnerton-Dyer congruences at $p$ as follows:

(1) If $p \equiv 1 \bmod 12$, then both $h_{1}$ and $h_{5}$ satisfy the 3-term Atkin and Swinnerton-Dyer congruences at $p$ with characteristic polynomial $T^{2}-\operatorname{sgn}(p) a(p) T+p^{2}$, where $\operatorname{sgn}(p)= \pm 1 \equiv$ $(-3)^{(p-1) / 4} \bmod p$;

(2) If $p \equiv 5 \bmod 12$, then $h_{1} \pm h_{5}$ satisfy the 3-term Atkin and Swinnerton-Dyer congruences at $p$ with characteristic polynomial $T^{2} \mp i a(p) T-p^{2}$

(3) If $p \equiv 7 \mathrm{mod} 12$, then both $h_{1}$ and $h_{5}$ satisfy the 3-term Atkin and Swinnerton-Dyer congruences at $p$ with characteristic polynomial $T^{2}-a(p) T-p^{2}$ and $T^{2}+a(p) T-p^{2}$ respectively;

(4) If $p \equiv 11 \bmod 12$ then $h_{1} \pm i h_{5}$ satisfy the 3-term Atkin and Swinnerton-Dyer congruences at $p$ with characteristic polynomial $T^{2} \mp a(p) T-p^{2}$.

Here $a(p)$ are the pth coefficients of $g^{\prime}(15)$.

\section{Acknowledgments}

The author is indebted to A.O.L. Atkin for his many original observations and insightful comments. The congruences stated in Theorem 20 were verified by Atkin computationally. The author would like to thank Wenching W. Li and Siu-Hung Ng for their continuous and valuable discussions and Chris A. Kurth for his comments on an earlier version of this paper. Special thanks are due to William A. Stein for computation assistance and the referee for many useful and constructive suggestions.

\section{References}

[ALL08] A.O.L. Atkin, W.C. Li, L. Long, On Atkin and Swinnerton-Dyer congruence relations (2), Math. Ann. 340 (2) (2008) 335-358.

[ASD71] A.O.L. Atkin, H.P.F. Swinnerton-Dyer, Modular forms on noncongruence subgroups, in: Combinatorics, Univ. California, Los Angeles, CA, 1968, in: Proc. Sympos. Pure Math., vol. XIX, Amer. Math. Soc., Providence, RI, 1971, pp. 1-25.

[Ber94] G. Berger, Hecke operators on noncongruence subgroups, C. R. Acad. Sci. Paris Sér. I Math. 319 (9) (1994) 915-919.

[Car71] P. Cartier, Groupes formels, fonctions automorphes et fonctions zeta des courbes elliptiques, in: Actes du Congrès International des Mathématiciens, Tome 2, Nice, 1970, Gauthier-Villars, Paris, 1971, pp. 291-299.

$\left[\mathrm{F}^{+} 05\right] \quad$ L. Fang, J.W. Hoffman, B. Linowitz, A. Rupinski, H. Verrill, Modular forms on noncongruence subgroups and Atkin-Swinnerton-Dyer relations, preprint, 2005.

[KL08] C.A. Kurth, L. Long, On modular forms for some noncongruence subgroups of $S L_{2}(\mathbb{Z})$, J. Number Theory (2008), doi:10.1016/j.jnt.2007.10.007.

[LLY05] W.C. Li, L. Long, Z. Yang, On Atkin and Swinnerton-Dyer congruence relations, J. Number Theory 113 (1) (2005) 117-148.

[Lon07] L. Long, Finite index subgroups of the modular group and their modular forms, arXiv:0707.3315, 2007.

[Ran67] R.A. Rankin, Lattice subgroups of free congruence groups, Invent. Math. 2 (1967) 215-221

[Sch85] A.J. Scholl, Modular forms and de Rham cohomology; Atkin-Swinnerton-Dyer congruences, Invent. Math. 79 (1) (1985) 49-77.

[Sch86] A.J. Scholl, Fourier coefficients of Eisenstein series on noncongruence subgroups, Math. Proc. Cambridge Philos. Soc. 99 (1) (1986) 11-17.

[Sch88] A.J. Scholl, The $l$-adic representations attached to a certain noncongruence subgroup, J. Reine Angew. Math. 392 (1988) 1-15. 
[Ser] J.P. Serre, Résumé de cours 1984-5, Collège de France.

[Ser77] Jean-Pierre Serre, Linear Representations of Finite Groups, Springer-Verlag, New York, 1977, translated from the second French edition by Leonard L. Scott, Grad. Texts in Math., vol. 42.

[Tho89] J.G. Thompson, Hecke operators and noncongruence subgroups, in: Group Theory, Singapore, 1987, de Gruyter, Berlin, 1989, pp. 215-224, including a letter from J.-P. Serre. 